\title{
Reliability of rail transverse flaw detection by means of an embedded ultrasonic based device
}

\author{
Hajar Benzeroual ${ }^{1, a}$, Abdellatif Khamlichi ${ }^{2}$ and Alia Zakriti $^{2}$ \\ ${ }^{1}$ Faculty of Sciences, Systems of Communication and Detection Laboratory, Department of Physics, 93000 Tetouan, Morocco \\ ${ }^{2}$ National School of Applied Sciences, Industrial and Civil Engineering Department, 93000 Tetouan, Morocco
}

\begin{abstract}
The main causes of train derailment are related to rail defects such as head surface defects, horizontal defects, vertical longitudinal split defects, star cracks at colt holes and diagonal crack in web. The most adverse form of these defects consists of transverse flaws that are either opened or internal. These flaws are developed generally in a plane which is orthogonal to the rail direction. Detection of these defects is essential in order to improve security of rail roads and to prevent catastrophic failures. Most of the inspection techniques of rails relay on eddy currents, electromagnetic induction and ultrasounds. Unlike some conventional inspection tests based on contact excitation probes that roll on the rail head, this work deals with theoretical analysis of an integrated contact-less system based on ultrasound waves. Generation of these waves was performed by using a non-ablative laser source. A rotational laser vibrometer was used to perform reception of the echoes. Through finite element modelling of the rail system under specific ultrasound excitation of the rail head, detection of transverse rail flaws was studied. Reliability of defect detection was then assessed by considering various sources of perturbations.
\end{abstract}

\section{Introduction}

Railways traffic is currently increasing and speed of modern trains is augmenting considerably. Maintenance of rails has become then a major challenge in order to assure sufficient safety of this mean of transportation to the occurrence of accidents. This task requires preventing rail failure which is known to yield catastrophic derailments with lots of casualties and huge economic loss [1]. Among the techniques used in the context of inspection of rails, the ultrasonic wave propagation based methods are very common [2].

Ultrasonic waves are waves having the form of very short pulses that are generated at the rail surface [3] and centred on a chosen work frequency. Usually, these frequencies range from $0.1 \mathrm{MHz}$ up to $50 \mathrm{MHz}$. Ultrasonic waves propagate in the rail structure under various dispersion patterns. When crossing an internal flaw, these waves produce echoes that can be sensed to determine some indications about the defect [4-7].

Some difficulties affect however, the capability of flaw detection within the context of ultrasound based techniques, as well as by using other known techniques. This happens especially when the defect is very small. The cause is mainly due to the presence of various sources of uncertainties and the involvement of some perturbations in the studied problems. Statistics have revealed in fact that almost $75 \%$ of predictions provided by actual inspection methods were false.
In ultrasonic based inspection techniques of railways, diffraction of the incident elastic waves occurs at any defect present in the rail system. The resulting echoes can then be sensed at some reception points by means of adequate implemented sensors. Excitation can be achieved by various methods. Here, focus is on irradiation of the rail by a laser beam source, while reception of the ultrasonic wave amplitudes is performed by a laser vibrometrer [8].

To explore the best configuration for transverse rail flaws detection as based on the previous mentioned approach, simulation of ultrasound waves crossing these defects is considered in the following. Specific ultrasound excitation of the rail head is performed. The finite element method through dynamic analysis under ABAQUS software packages is used. Detection is based on change of wave signal indicator between the two rail situations: (1) the undamaged rail taken to be the reference state and (2) the rail state in the course of inspection with the presence of a localized transverse crack.

Different crack depths are considered in this study. Reliability of defect detection is assessed by considering sources of perturbations related to rail material properties and laser irradiation spot. Analysis of Variance (ANOVA) is introduced to determine the most influential factor and to analyze sensitivity of the indicator to variations of the intervening factors.

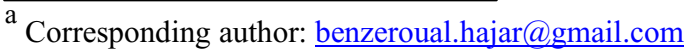




\section{Modelling methods and reliability analysis}

\subsection{Modelling propagation of ultrasonic waves in rail system}

A finite element model of the rail is proposed in the following. Simulation of ultrasonic elastic wave propagation in the European standard rail structure 60E1 is considered. Figure 1 shows the geometry of the rail system with a localized defect having the shape of a transverse crack.

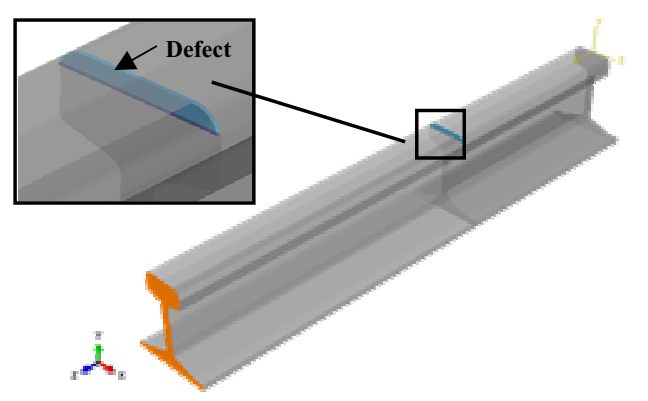

Figure 1. Schematic of a rail with localized transverse flaw.

Rail structure is assumed to be made from linear homogeneous and isotropic elastic steel material. The mechanical properties are given in Table 1. The modelled portion of the rail has length $1.3 \mathrm{~mm}$. The geometry of the defect is rectangular and has $10 \mathrm{~mm}$ in depth and $2 \mathrm{~mm}$ in width.

Table 1. Mechanical properties of the considered rail.

\begin{tabular}{|c|c|}
\hline Young's Modulus $(\mathrm{GPa})$ & 210 \\
\hline Density $\left(\mathrm{kg} / \mathrm{m}^{3}\right)$ & 7800 \\
\hline Poisson's ratio & 0.3 \\
\hline Conductivity $(\mathrm{W} / \mathrm{m} / \mathrm{K})$ & 50.2 \\
\hline
\end{tabular}

The method of excitation is based on laser generated ultrasound. This technique is a contact-less arrangement that enables to generate ultrasonic signals remotely with the aid of lasers [9]. Short and focused laser excitation is performed on the top head of the rail to excite a small spot of the surface of the rail and to generate ultrasonic waves. In this case the spot size is chosen to be $s=0.015 \mathrm{~m}$. Figure 2 shows such a configuration.

For reception of wave signals, a sensor using laser vibrometry concept is used to measure the elastic rotation occurring at any given section of the rail. This sensor is termed Rotational Laser Vibrometer (RLV). It enables to detect the reflected energy of the transmitted beam. It is also a contact-less device that allows measuring very accurately the angular velocity and angular displacement generated by elastic deformation between any pair of points on the rail structure. In this work, the RLV is calibrated to measure the normal velocity to the head surface of the rail, velocity in the ydirection $\left(V_{y}\right.$ in $\left.\mathrm{m} / \mathrm{s}\right)$. The sensor works with a given pair of points. Figure 3 gives the configuration of some points selected in order to measure the deformation caused by propagating ultrasonic waves.

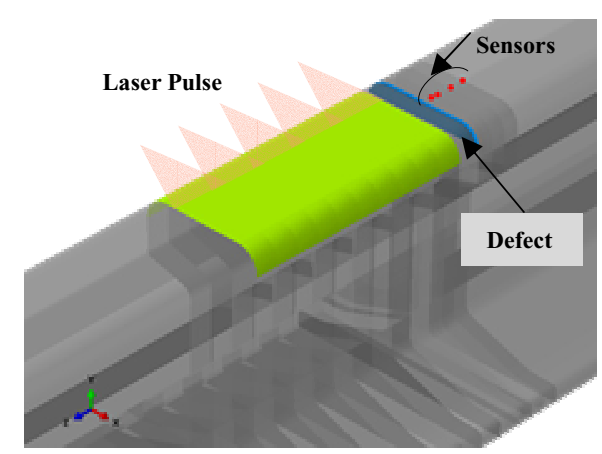

Figure 2. Position of sensors on the rail with the laser spot.

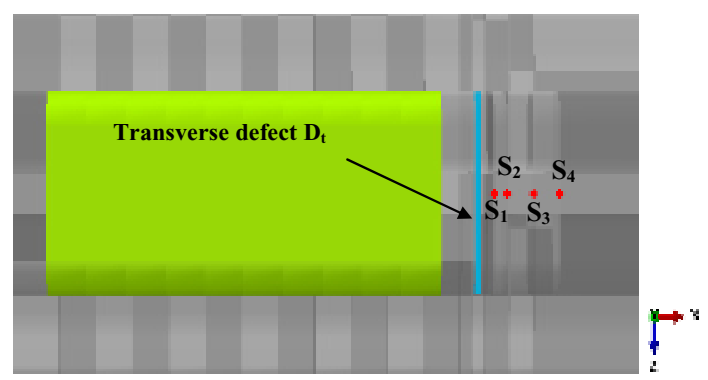

Figure 3. Position of sensors $\left(S_{i}\right)$ and the defect $\left(D_{t}\right)$.

\subsection{Simulation of ultrasonic waves}

The excitation pulse can be generated by modulation of laser spot irradiation [6]. Here, the pulse duration was fixed at $\Delta T=450 \mu \mathrm{s}$ and a train of 20 sequential steps of the same duration was applied. The total time of computation is $100 \Delta T$. The $V_{y}$ amplitude obtained at each sensor location at the final instant $t_{f}=45 \mathrm{~ms}$ are the main information used in detection.

In order to evaluate the influence of the various parameters on the rail response, in the presence of a transverse defect, simulations were carried out according to all combinations resulting from the design levels that are indicated in Table 2. The chosen parameters include: laser spot area $s$, depth of the crack $d$ and Young modulus $E$.

Table 2. Levels of the considered parameters.

\begin{tabular}{|c|c|c|c|}
\hline Parameters & $s(\mathrm{~mm})$ & $d(\mathrm{~mm})$ & $E(G P a)$ \\
\hline Level 1 & 14.85 & 9.9 & 208 \\
\hline Level 2 & 15 & 10 & 210 \\
\hline
\end{tabular}

Simulations were performed for both intact and defected rail. The considered combinations served to evaluate the relative influence of the selected factors. 
Standard ANOVA procedure was used to analyze variability of the sensed wave intensity as function of variations of factors.

\subsection{Reliability analysis of flaw detection}

Structures are always subjected to uncertainties due to geometric variations, stochastic material properties and applied loading. These make their response to be random and circumstances under which they manifest some performance criterion are therefore not deterministic. Reliability is a pertinent tool that enables to quantify the effects of these uncertainties and to calculate the probability of failure for a given performance function. This is achieved by performing propagation of the uncertainties from the densities of probabilities of the random variables which are present at the basic level as inputs of the system to the considered performance criterion which is the actual output [10].

The performance criterion called also limit state is here related to the aptitude of detecting a transverse flaw which is present in the rail head with given characteristics. Failure of detection is considered to occur when the following condition is satisfied

$$
g(X) \leq 0
$$

where $X$ designates a vector containing all the variables that are involved in the reliability analysis and $g$ is the limit state function which separates the acceptable region with affirmative detection from that corresponding to failure of detection.

In the present case, the performance function cannot be obtained explicitly. Coupling software that predicts the actual state of the system with a reliability code is a method that can be used to perform reliability analysis. However, this process is costly [10]. There exist various methods that can be used to get a fast and robust approximation of the performance function if it is implicitly determinate. These methods enable to construct an explicit mathematical representation of the exact limit state and permit its direct evaluation as function of the basic random variables of the process.

The explicit representation model of the system is constructed by performing at first an adequate choice of trial points over the fixed domain to be explored as function of the basic variables. This is generally performed according to a design of experiment table based procedure. The simplest approach to select trial points uses a full factorial table that is generated from the selected discrete levels of the factors [11]. Using the results of simulations for these particular points, a metamodel can be derived. This provides an explicit representation of the state function over the considered domain of basic variables.

A common approach that is generally used to derive a metamodel of the system is the Response Surface Method (RSM) based method. The RSM takes usually the form of a polynomial function. Defining the densities of probabilities that describe the intervening random variables permits then to use Monte Carlo Simulation (MCS) to calculate the probability of failure.
The MCS approach enables to generate values of a random variable at the output based the probability distributions that describe variability of the inputs. By increasing the number of samples, the estimated probability of failure converges. By denoting $n_{f}$ the obtained total number of failure realizations, the failure probability $p_{f}$ can be estimated as

$$
p_{f}=n_{f} / N
$$

where $N$ is the total number of samples. The coefficient of variation of the estimate $p_{f}$ is proportional to $1 / \sqrt{N}$. This means that in order to achieve an estimate of $p_{f}$ in the order of 0.001 , approximately $N=10^{5}$ simulations are required.

\section{Results and discussion}

Considering the rail system described in section 2.1, simulations have been performed under ABAQUS for all the combinations resulting from a full factorial design of experiment table constructed on Table 2. For each combination, the amplitude of y-velocity at sensors locations $\left(S_{i}, i=1,2,3,4\right)$, as shown in Figure 3, was obtained at the time instant $t_{f}=45 \mathrm{~ms}$. This was performed for both defect free rail and rail having a transverse like defect. Table 3 gives the obtained results for a rail with defect, while Table 4 gives the results for a defect free rail.

Table 3. The results of sensor 1 and 2 in $(\mathrm{mm} / \mathrm{s})$, for the case of rail with transverse defect.

\begin{tabular}{|c|c|c|c|c|c|c|}
\cline { 3 - 7 } \multicolumn{1}{c}{} & \multicolumn{5}{c}{ With transverse defect } \\
\hline $\mathrm{s}(\mathrm{mm})$ & $\mathrm{d}(\mathrm{mm})$ & $E(\mathrm{GPa})$ & $S_{1}$ & $S_{2}$ & $S_{3}$ & $S_{4}$ \\
\hline 15 & 10 & 210 & 1.53 & 1.46 & 1.24 & 1.12 \\
\hline 15 & 10 & 208 & 1.44 & 1.39 & 1.15 & 1.01 \\
\hline 15 & 9.9 & 210 & 1.47 & 1.42 & 1.34 & 1.09 \\
\hline 15 & 9.9 & 208 & 1.44 & 1.41 & 1.32 & 1.06 \\
\hline 14.85 & 10 & 210 & 1.53 & 1.47 & 1.31 & 1.23 \\
\hline 14.85 & 10 & 208 & 1.44 & 1.39 & 1.22 & 1.14 \\
\hline 14.85 & 9.9 & 210 & 1.51 & 1.48 & 1.30 & 1.09 \\
\hline 14.85 & 9.9 & 208 & 1.49 & 1.46 & 1.27 & 1.07 \\
\hline
\end{tabular}

Table 4. The results of sensors in $(\mathrm{mm} / \mathrm{s})$, for the case of defect- free rail.

\begin{tabular}{|c|c|c|c|c|c|}
\cline { 3 - 6 } \multicolumn{2}{c}{} & \multicolumn{5}{c}{ Without transverse defect } \\
\hline $\mathrm{s}(\mathrm{mm})$ & $E(\mathrm{GPa})$ & $S_{1}$ & $S_{2}$ & $S_{3}$ & $S_{4}$ \\
\hline 15 & 210 & 1.98 & 1.93 & 1.72 & 1.41 \\
\hline 15 & 208 & 1.87 & 1.84 & 1.61 & 1.27 \\
\hline 14.85 & 210 & 2.01 & 1.99 & 1.74 & 1.46 \\
\hline 14.85 & 208 & 1.94 & 1.91 & 1.67 & 1.32 \\
\hline
\end{tabular}

To perform detection, the difference between the values at two sensor locations for a rail with a defect and a defect free rail were calculated. Detection is based on variation of these amplitudes as the device crosses a defected zone. It is interesting to analyze at first how 
these signals vary as function of the input data: spot size, defect depth and Young modulus. Figure 4 gives the results of ANOVA performed on the obtained amplitudes as calculated from data given in Table 3.

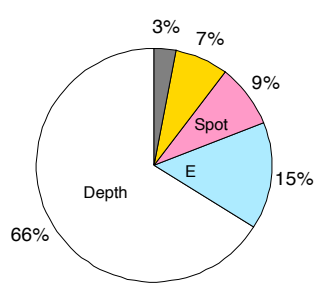

$S 1-S 2$

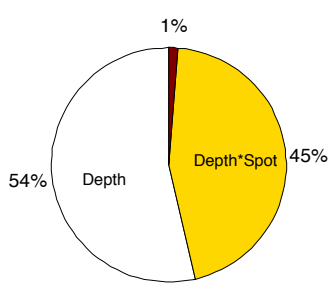

S1-S3

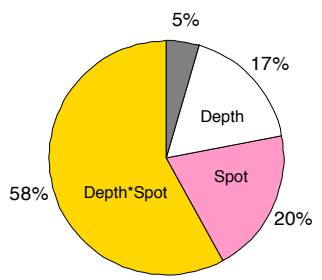

$S 1-S 4$

Figure 4. ANOVA results for the pair of sensors $S 1-S 2, S 1-S 3$ and S1-S4; rail with a defect

Figure 4 shows that $S 1-S 2$ configuration is sensitive to Young modulus and spot size even if it is more sensitive to defect depth than the others. The third case $S 1-S 4$ is also sensitive to spot size. ANOVA indicates that the best configuration is provided by sensors $S 1-S 3$ as sensitivity to crack depth is higher and effect of perturbations is reduced.

To analyze reliability of detection based on variation of the amplitudes while traversing a defect, a RSM was derived for the output associated to defect free rail and rail with a defect. The obtained regressions have all high coefficient of determination which was superior to $99 \%$. As an example, the obtained amplitudes for respectively rail with defect and defect free rail for sensors $S 1-S 3$ are:

$$
\begin{aligned}
A_{13}= & -1145.8+1220.2 s+1077.3 d+10.751 E \\
& -1152.3 s d-73.493 s E+63.25 d E \\
A_{13}^{0}= & -31.193-32.081 s-31.532 E+32.424 s E
\end{aligned}
$$

Monte-Carlo simulations were performed in Matlab for the three configurations of sensors. Figure 5 gives the obtained results corresponding to the basic variables modeled by means of the lognormal distribution for spot size and crack depth, while the Young modulus variations were distributed according to a Gaussian law. All these distributions of probabilities were centered on the median values given in Table 3 and were affected by $10 \%$ standard variation. The circles in Figure 5 indicate the maximum amplitude obtained by the considered pair of sensors while crossing a defect.

It is interesting to see that sensors configurations $S 1$ $S 3$ necessitates the least resolution, while the S1-S2 could not work without sensors of ultra-high resolution as the maximal signal amplitude represented by the red circle is too small.

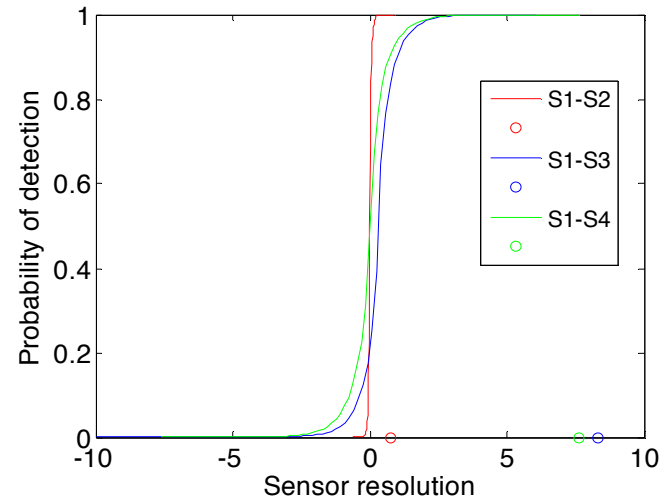

Figure 5. Probability of detection as function of the sensor resolution for the three configurations $S 1-S 2, S 1-S 3$ and $S 1-S 4$

\section{Conclusions}

The main point revealed at this level of the study is the influence of the distance separating the sensor from the defect. Each sensor captures information depending on this distance and once the distance changes, the influence of factors (the spot and depth of the defect in particular) changes also. The obtained results have enabled to select the best configuration of sensors locations in order to operate detection of rail crack with high resolution and reduced sensitivity to uncertainties on spot size and material Young modulus.

\section{References}

1. D.F. Cannon, K.O. Edel, S.L. Grassie, K. Sawley, Fatigue and Fracture of Engineering Materials and Structures 26, 10 (2003)

2. G. Zumpano, M. Meo, International Journal of Solids and Structures 43, 5 (2006)

3. U. Zerbst, R. Lundén, K.O. Edel, R.A. Smith, Engineering Fracture Mechanics 76, 17 (2009)

4. R. Clark, NDT\&E International 37, 2 (2004)

5. J.J. Marais and K.C. Mistry, Fatigue and Fracture of Engineering Materials and Structures 26, 10 (2003)

6. S. Teidj S., A. Khamlichi, A. Driouach, E. Jacquelin, International Journal of Railway Technology 3, 3 (2014)

7. J.L. Rose, M.J. Avioli, P. Mudge, R. Sanderson, NDT\&E International 37, 2 (2004)

8. S.J. Rothberg, M. Tirabassi, Mechanical Systems and Signal Processing 26 (2012)

9. R. J. Dewhurst and Q. Shan, Optical remote measurement of ultrasound, Meas. Sci. Technol. 10, 11 (1999)

10. O. Ditlevsen, H.O. Madsen, Structural reliability methods, John Wiley and Sons Hoboken (1996)

11. W.J. Roux, N. Stander, R.T. Haftka, Journal for Numerical Methods in Engineering 42, 3 (1998) 